\title{
The Neuraminidase Inhibitor Peramivir Ameliorates Myocarditis Induced by Influenza A (H1N1pdm) Virus in a Murine Model
}

\author{
Akira Ukimura $^{1,2}$, Yukimasa $0 i^{2,3}$ and Yumiko Kanzaki ${ }^{3}$ \\ ${ }^{1}$ Department of General Internal Medicine, Osaka Medical College, Takatsuki, Japan \\ ${ }^{2}$ Infection Control Team, Osaka Medical College Hospital, Takatsuki, Japan \\ ${ }^{3}$ Third Department of Internal Medicine, Osaka Medical College, Takatsuki, Japan
}

Corresponding author: Akira Ukimura, MD, PhD, Department of General Internal Medicine, Osaka Medical College, Takatsuki, 569-8686, Japan, Tel: +81-72-683-1221; Fax: +81-72-684-7386; E-mail: in3011@poh.osaka-med.ac.jp

Received date: March 13, 2015; Accepted date: May 21, 2015; Published date: May 28, 2015

Copyright: (C) 2015 Ukimura A, et al. This is an open-access article distributed under the terms of the Creative Commons Attribution License, which permits unrestricted use, distribution, and reproduction in any medium, provided the original author and source are credited.

\begin{abstract}
Severe influenza sometimes causes myocarditis. To analyze the effects of peramivir on influenza A (H1N1pdm) virus myocarditis, we investigated survival rates, cardiac function, histological findings and cytokine induction in murine influenza A (H1N1pdm) virus-induced myocarditis. Eight-week-old BALB/c male mice were infected intranasally with influenza $A(\mathrm{H} 1 \mathrm{~N} 1 \mathrm{pdm})$ virus, and then divided into 2 groups: control group, which was injected with saline; and peramivir-treatment group, which was treated with peramivir. Histological studies, echocardiograms and quantitative analysis of viral RNA and mRNA for inflammatory cytokines and adhesion molecules were performed. Treatment with peramivir led to a significant improvement in survival $(p<0.01$ vs. control group). Fractional shortening (FS) of peramivir-treatment group $(44.7 \%, p<0.01)$ was significantly higher than that of control group $(27.7 \%)$ on day 8 . Histological examinations revealed localized myocarditis with lymphocyte infiltration, and myocarditis lesions were found in perivascular areas or associated with pericarditis; treatment with peramivir improved these findings. Quantity of influenza virus genome in heart and lung tissues was suppressed in peramivirtreatment group. The expression of cytokine and adhesion molecule mRNA was suppressed in peramivir-treatment group. Peramivir improved influenza A (H1N1pdm) virus-induced myocarditis.
\end{abstract}

Keywords: Myocarditis; Influenza; Peramivir

\section{Introduction}

Acute myocarditis is a potentially lethal disease; etiological agents include Enteroviruses, Adenoviruses, Parvoviruses, Cytomegalovirus, Influenza virus and others [1-6]. Fulminant myocarditis causes severe hemodynamic dysfunction and requires high-dose catecholamine and mechanical circulatory support [1,6-8]. An influenza pandemic occurred in $2009[6,9,10]$. The causative organism, influenza A (H1N1pdm) virus, has been reported to cause fatal myocarditis as well as pneumonia [11-14]. Based on national surveillance in Japan, we previously reported that 19 fulminant myocarditis patients (adults: 13, children: 6) with influenza A (H1N1pdm) virus were seen in the 2009/2010 season, while only 2 adult patients were seen in the 2010/2011 season [12-14].

The course of cardiac dysfunction and recommended timing of interventions are described in the guidelines for diagnosis and treatment of myocarditis of the Japan Circulation Society (JCS2009). Myocarditis is treated in three ways: (1) intervention to eliminate the cause, (2) intervention to improve hemodynamic compromise, and (3) intervention to address cardiac dysfunction $[1,6,8]$. In addition, not only viral myocarditis but also other infections by various microbials in various animals are exacerbated by non-steroidal anti-inflammatory drugs (NSAIDs) use $[15,16]$. Intervention to avoid exacerbating cause such as NSAIDs including salicylates may reduce serious complication including myocarditis.
The neuraminidase inhibitors represent an important advance in the treatment of influenza [17-24]. Treatment with neuraminidase inhibitors is also recommended by the Japanese association of Infection for all patients infected with influenza [25,26]. Sugaya reported Japan may have had the lowest case fatality rate for symptomatic illness $(<0.001 \%, 198 / 20.7$ million $)$ in the H1N1 pandemic because of the universal implementation of early treatment with neuraminidase inhibitor [21]. On the other hand, Hama et al. reported oseltamivir could induce sudden deterioration leading to death with 12 hours of prescription [27]. Effects of neuraminidase inhibitors on mortality seemed to be controversial.

It was also difficult to show that the neuraminidase inhibitors significantly improved the survival rate of patients with fulminant myocarditis associated with influenza [6,7,11-14]. Peramivir is a selective inhibitor of influenza neuraminidase that is effective when administrated intramuscularly and intravenously for the treatment of influenza virus infection in mouse models [17-20,23]. To analyze the effects of peramivir on influenza A (H1N1pdm) virus myocarditis, we investigated survival rates, cardiac function, histological findings and cytokine induction in murine influenza A (H1N1pdm) virus -induced myocarditis.

\section{Materials and Methods}

\section{Cell culture and virus}

The influenza A (H1N1pdm) virus (IAV) used in this study was obtained from the lung of a patient who died of IAV pneumonia in 2009 in Japan (A/Niigata/09F098/2009). IAV was plaque-purified in 
Citation: Ukimura A, Ooi Y, Kanzaki Y (2015) The Neuraminidase Inhibitor Peramivir Ameliorates Myocarditis Induced by Influenza A (H1N1pdm) Virus in a Murine Model. Clin Microbiol 4: 201. doi:10.4172/2327-5073.1000201

Page 2 of 5

Madin-Darby canine kidney (MDCK) cells, and passaged three times in MDCK cells. The virus fluids were stocked at $-80^{\circ} \mathrm{C}$ until used.

\section{Animal experimentation}

Specific pathogen-free 8-week-old male BALB/c mice weighing 16 g-20 g each were obtained from SLC laboratories (Shizuoka, Japan). Mice were permitted an acclimatization period of greater than $48 \mathrm{~h}$ prior to virus inoculation, during which time the animals were observed for signs of disease and/or physical abnormalities. Peramivir trihydrate was obtained from shionogi pharmaceuticals (Osaka, Japan).

The 8-week-old male BALB/c mice were infected intra-nasally with 50 plaque-forming units of IAV under anesthesia, and then were divided into 2 groups: control group (group C), which was injected with saline as a vehicle; and peramivir-treatment group (group P), which was treated with peramivir at $50 \mathrm{mg} / \mathrm{kg}$ by intramuscular injection daily for 3 days after virus inoculation. There were 20 mice in each group, and we used another 30 mice for echocardiographic study and histological study in control group and peramivir-treatment group. Uninfected mice $(n=20)$ treated with saline were used for the uninfected group (group $\mathrm{U}$ ).

To assess cardiac function, an echocardiograph was performed with a $12 \mathrm{MHz}$ transducer on an aplio ultrasound machine (Toshiba medical systems, Tochigi, Japan) under controlled anesthesia on postinfection day 8. Left ventricular end-diastolic dimension (LVeDD) and left ventricular end-systolic dimension (LVeSD) and the percent fractional shortening (FS), which was calculated by the formula $[(\mathrm{LVeDD}-\mathrm{LVeSD} / \mathrm{LVeDD}) \times 100]$, were measured from five consecutive cycles and averaged.

In each group, 6 mice were killed on days 5 and 8, after anesthesia administration, and the hearts were cross-sectioned across both ventricles after measurement of body weight and heart weight. Heart weight $(\mathrm{mg})$ /body weight $(\mathrm{mg})(\mathrm{HW} / \mathrm{BW})$ ratio was calculated. Half of each heart and lung was fixed in $10 \%(\mathrm{v} / \mathrm{v})$ buffered formalin and used for histology by staining with hematoxylin and eosin. The other half of each heart and lung was frozen in liquid nitrogen and used for subsequent PCR analysis. This study protocol conformed to the guide for the care and use of laboratory animals (NIH Publication No. 85-23, 1996) and was approved by the animal care committee of Osaka medical college.

\section{Quantitative real-time polymerase chain reaction analysis}

Total RNA was extracted from myocardial tissues and lung with an RNeasy mint kit (Qiagen, Tokyo, Japan), and reverse transcription was performed with random hexamers and reverse transcriptase. Quantitative PCR was performed using a Step One system (Applied Biosystems, Drive Foster, CA, USA). The reaction conditions were as follows: $50^{\circ} \mathrm{C}$ for $2 \mathrm{~min}, 95^{\circ} \mathrm{C}$ for $10 \mathrm{~min}$, and then 40 cycles of $95^{\circ} \mathrm{C}$ for $15 \mathrm{sec}$ and $60^{\circ} \mathrm{C}$ for $1 \mathrm{~min}$. The relative quantity of each target mRNA [IAV genome, brain natriuretic peptide (BNP), interferon- $\gamma$ (IFN-G), interleukin-6 (IL-6), and vascular cell adhesion molecule-1 (VCAM-1)] was evaluated by comparison to glyceraldehyde-3phosphate dehydrogenase (GAPDH) mRNA expression. We used pairs of primers and TaqMan probes for step one system purchased from applied biosystems for IFN-G, IL-6, VCAM-1 and BNP. We used primers of neuraminidase segments of influenza virus for quantitative RT-PCR.

\section{Statistical analysis}

Results are presented as mean $\pm \mathrm{SD}$. One-way analysis of variance (ANOVA) was performed to evaluate differences among the 3 groups. Wilcoxon's two-rank-sum test (Mann-Whitney U test) was used to evaluate differences between 2 groups. Kaplan-Meier survival analysis was applied to assess survival number differences. $\mathrm{P}$ values of $<0.05$ were considered statistically significant.

\section{Results}

\section{Mortality and histological findings}

Treatment with peramivir resulted in significant improvement in survival ( $<<0.01$ vs. control group) (Figure 1). Body weight (17.9 $\mathrm{g} \pm$ $0.6 \mathrm{~g}$ ) of control group was significantly smaller than peramivirtreatment group $(19.7 \mathrm{~g} \pm 0.6 \mathrm{~g})(\mathrm{p}<0.01)$, and HW/BW ratio $(6.1 \pm$ 0.4 ) was significant higher than peramivir-treatment group (5.5 \pm 0.2$)$ $(\mathrm{p}<0.01)$ on day 8 (Figure 1$)$. Mice intra-nasally inoculated with IAV exhibited acute pneumonia and myocarditis, as confirmed by histological examination of hematoxylin and eosin-stained sections. Histological findings showed myocyte degeneration with lymphocyte infiltration and interstitial edema associated with pericarditis, and myocarditis lesions were also found in the perivascular area (Figure $2 \mathrm{~A}-2 \mathrm{C}$ ), and mononuclear cells infiltrated in lung in control group (Figure 2E). However, histological studies showed localized myocarditis with lymphocyte infiltration. Peramivir ameliorated these histopathological findings (Figures $2 \mathrm{D}$ and $2 \mathrm{~F}$ ).

Figure 1.

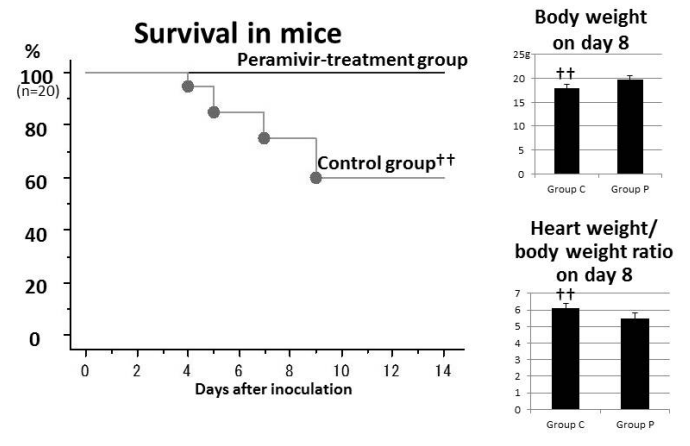

Figure 1: Effects of peramivir on survival, body weight and heart weight/body weight ratio in mice infected with Influenza $A$ virus ; we used 20 mice in each group in the survival study. $(100 \%: \mathrm{n}=20)$. ${ }^{\dagger \dagger} \mathrm{p}<0.01$, peramivir-treatment group vs. control group.

\section{Echocardiographic findings}

Echocardiograms revealed left ventricular dilatation and left ventricular systolic dysfunction in group V. The LVeDD in peramivirtreatment group $(3.1 \mathrm{~mm})$ was significantly smaller than in control group $(3.4 \mathrm{~mm})$ on day $8(\mathrm{p}<0.05)$. The FS of uninfected group $(51.5 \%$, $\mathrm{p}<0.0001)$ and peramivir-treatment group $(44.6 \%, \mathrm{p}<0.001)$ was 
Citation: Ukimura A, Ooi Y, Kanzaki Y (2015) The Neuraminidase Inhibitor Peramivir Ameliorates Myocarditis Induced by Influenza A (H1N1pdm) Virus in a Murine Model. Clin Microbiol 4: 201. doi:10.4172/2327-5073.1000201

Page 3 of 5

significantly greater than the FS of control group (27.7\%) on day 8 (Figure 3).

Figure 2.

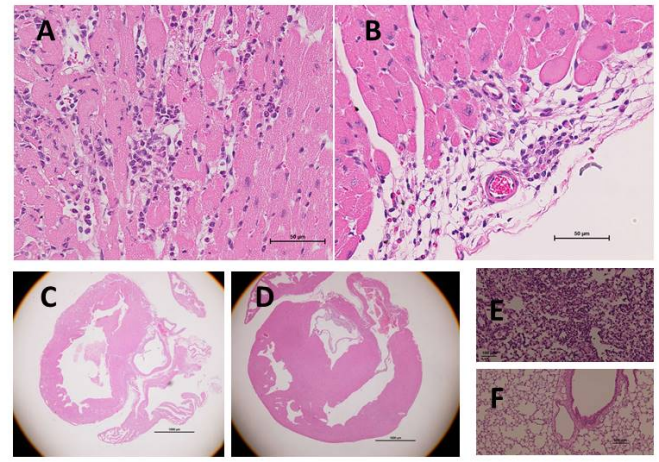

Figure 2: Histological analysis of the effects of peramivir on the development of myocarditis. Cross sections of the heart of mice in $(\mathrm{A}, \mathrm{B}, \mathrm{C})$ the control group and (D) the peramivir-treatment group stained with hematoxylin and eosin on study day 5 . Histological findings showed myocyte degeneration with lymphocyte infiltration and interstitial edema associated with pericarditis, and myocarditis lesions were also found in the perivascular area in control group. Cross sections of the lung of mice in (E) the control group and $(F)$ the peramivir-treatment group stained with hematoxylin and eosin on study day 5. Histological findings showed mononuclear cells infiltration in lung in control group. Scale bar: $50 \mu \mathrm{m}$ in $\mathrm{A}$ and $\mathrm{B}, 1,000 \mu \mathrm{m}$ in $\mathrm{C}$ and $\mathrm{D}, 100 \mu \mathrm{m}$ in $\mathrm{E}$ and F.

\section{Quantitative real-time polymerase chain reaction analysis}

The relative expressions of IAV genome and pro-inflammatory cytokines, BNP and VCAM-1, are summarized in Figure 4. Peramivir treatment significantly decreased quantity of IAV genome in the heart and lung tissues on day 5, compared with control group (Figure 4), and significantly decreased the expression of BNP in the myocardium on day 8, compared with control group. Compared with control group, expression of IFN-G, IL-6, and VCAM-1 mRNA was suppressed in peramivir-treatment group. However there was no significant difference of IL-6 in heart tissue between control group and peramivir-treatment group. There was no significant difference of IFN-G or VCAM-1 in lung tissue between control group and peramivir-treatment group.

\section{Discussion}

This study demonstrated the effect of peramivir treatment on influenza viral myocarditis in a mouse model. Treatment with peramivir improved the survival rate, histological findings and echocardiographic findings with suppression of quantity of IAV genome and BNP mRNA expression in this paper.

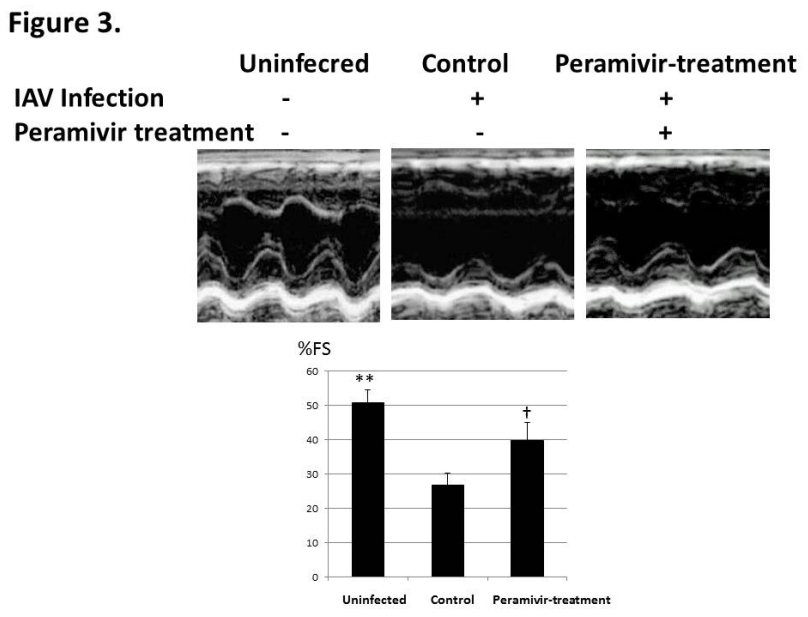

Figure 3: Echocardiograms show the suppression of cardiac function by Influenza $A$ virus (IAV) infection and its improvement by peramivir. Representative M-mode echocardiogram images of mice infected with IAV with or without intramuscular administration of peramivir at $50 \mathrm{mg} / \mathrm{kg}$ once daily for 3 days. Measurements of left ventricular end-diastolic dimension (LVeDD) and $\%$ fractional shortening (FS) were performed on day $8 .{ }^{\dagger} \mathrm{p}<0.05$, peramivir-treatment group vs. control group. ${ }^{* *} \mathrm{P}<0.01$, unincected group vs. control group.

Figure 4.

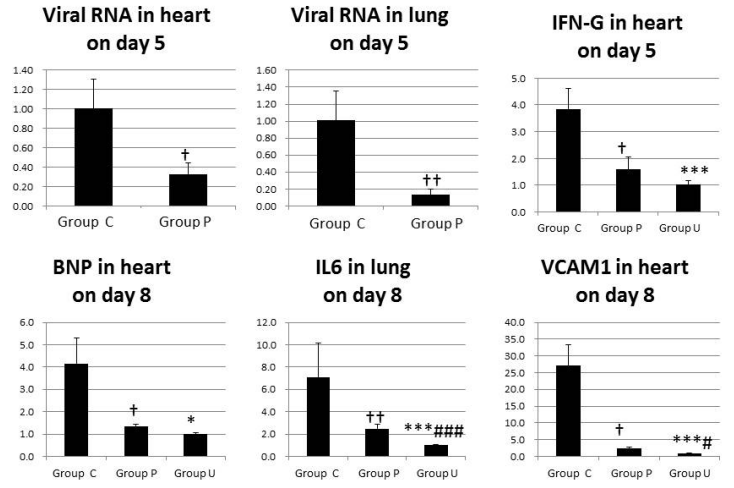

Figure 4: Relative expression of the Influenza $A$ virus (IAV) genome in heart or lung tissue by RT-PCR. Relative expression (corrected by GAPDH expression) of target mRNA in heart or lung tissues by RT-PCR. Columns and bars: mean $\pm \mathrm{SD} .{ }^{\dagger} \mathrm{p}<0.05$, peramivir-treatment group (P) vs. control group (C). ${ }^{\dagger \dagger} \mathrm{p}<0.01$, peramivir-treatment group $(\mathrm{P})$ vs. control group $(\mathrm{C}) .{ }^{*} \mathrm{P}<0.05$, unincected group (U) vs. control group $(\mathrm{C}) .{ }^{* * *} \mathrm{P}<0.001$, unincected group (U) vs. control group (C). ${ }^{\#} \mathrm{P}<0.05$, unincected group (U) vs. peramivir-treatment group $(\mathrm{P}) .{ }^{\# \# \#} \mathrm{P}<0.001$, unincected group $(\mathrm{U})$ vs. peramivir-treatment group (P). interferon- $\gamma$ (IFN-G), brain natriuretic peptide (BNP), interleukin-6 (IL-6), and vascular cell adhesion molecule-1 (VCAM-1). 
Peramivir is one of a selective inhibitor of neuraminidase of influenza virus [17,19]. It was reported that peramivir increased survival rate of animals infected with lethal dose of influenza virus by the pharmaceutical company of peramivir, and its in vitro activity has been demonstrated to be comparable to or better than that of oseltamivir carboxylate and zanamivir $[17,28]$. Peramivir has been approved in Japan for the treatment of general seasonal influenza in adults, as a single dose of $300 \mathrm{mg}$ intravenously [18,21]. A single dose or multiple daily doses of $600 \mathrm{mg}$ of peramivir are administered intravenously depending on the condition of the patient. We used the $50 \mathrm{mg} / \mathrm{kg}$ dose in mice for 3 days, which is approximately a humanequivalent dose of $300 \mathrm{mg}$ [19]. We showed suppression of quantity of IAV genome in this study. However, we did not analyze myocardial virus titer or neutralizing antibody titers, which is one of the limitations of this study [29-31]. Pan et al reported the influenza viral levels in the hearts monitored by the nonstructural protein 1 gene and nucleoprotein was in peak on day 6 [32]. However, there is no evidence of viable virus in this paper.

Influenza is a recognized cause of myocarditis, which can lead to significant impairment of cardiac function and mortality $[2,3,6]$. Myocarditis is not the major cause of death from influenza infection, and is very rare. However the number of influenza myocarditis increased in the H1N1 pandemic, and it was serious problem for cardiologists [12,13]. The pathological effects of influenza viral myocarditis in humans and mice are reportedly milder and more localized than those seen in coxsackievirus myocarditis [32-35]. In this study, histological findings showed localized myocarditis with lymphocyte infiltration, and myocarditis lesions were found in the perivascular area, or myocarditis was associated with pericarditis. Peramivir ameliorated these histopathological findings. Kotaka et al. reported that murine influenza myocarditis was histologically mild and brief in duration compared to coxsackievirus B3 myocarditis, and electron microscopic findings of the heart from a murine influenza myocarditis model showed many infiltrating lymphocytes directly attached to the cardiac myocytes [33]. Pan et al. investigated the molecular mechanism of influenza virus-associated myocarditis and revealed the importance of trypsin induction and the increased production of matrix metalloproteinase and proinflammatory cytokines in the pathogenesis of acute myocarditis [32,35]. Along with the direct effect of influenza virus infection, proinflammatory cytokines and endothelial cell dysfunction are thought to contribute to the pathogenesis of severe clinical features, including severe cardiac dysfunction, in patients infected with influenza virus [2,3,6,32,35].It was reported that osertamivir has pleotropic effects [30,36-38]. In human, compared with the partial reduction of viral shedding, proinflammatory cytokines are completely suppressed by oseltamivir use [36]. Oseltamivir reduced cellular response in the BALF and proinflammatory cytokines without reducing viral load in the lung if administered to mice infected with mild influenza virus [30]. Moore et al reported administration of oseltamivir in animals challenged by respiratory syncytial virus that lacks a neuraminidase gene showed a symptom-relieving effect and inhibition of viral clearance [38]. Evidence of pleotropic effects of peramivir is poor. Tanaka et al reported that a multiple-dose regimen of intravenous peramivir was more efficacious than a single peramivir dose or multiple doses of oseltamivir for improving outcomes in pneumococcal pneumonia following influenza virus infection in mice, and the production of inflammatory cytokines/chemokines was also significantly suppressed by multiple dosing of peramivir compared with oseltamivir. [29] Expression of IFN-G, IL-6, and VCAM-1 mRNA was suppressed by peramivir treatment in this study. Peramivir may have potentiality to suppress pro-inflammatory cytokines.

We previously reported that the frequency of cardiac involvement is likely elevated in influenza A (H1N1pdm) virus infection compared to seasonal influenza infection, according to results of national surveillance in Japan [12-14]. The myocardial toxigenicity of the seasonal influenza virus seems to be rather weak, so we used the pandemic virus obtained from the lung of a patient who died of IAV pneumonia in this study.

In conclusion, peramivir improved influenza A (H1N1pdm) virusinduced murine myocarditis.

\section{Conflict of Interest}

All authors declare that there are no conflicts of interest.

\section{Acknowledgement}

This study was supported in part by a research grant for intractable diseases from the Ministry of Health, Labour and Welfare, and a grant for scientific research from the Ministry of Education, Science and Culture, Tokyo, Japan. The authors greatly appreciate the excellent assistance of Ms. M. Kobayashi.

\section{References}

1. JCS Joint Working Group (2011) Guidelines for diagnosis and treatment of myocarditis (JCS 2009): digest version. Circ J 75: 734-743.

2. Estabragh ZR, Mamas MA (2013) The cardiovascular manifestations of influenza: a systematic review. Int J Cardiol 167: 2397-2403.

3. Mamas MA, Fraser D, Neyses L (2008) Cardiovascular manifestations associated with influenza virus infection. Int J Cardiol 130: 304-309.

4. Bowles NE, Ni J, Kearney DL, Pauschinger M, Schultheiss HP, et al. (2003) Detection of viruses in myocardial tissues by polymerase chain reaction. evidence of adenovirus as a common cause of myocarditis in children and adults. J Am Coll Cardiol 42: 466-472.

5. Koide H, Kitaura Y, Deguchi H, Ukimura A, Kawamura K, et al. (1992) Genomic detection of enteroviruses in the myocardium--studies on animal hearts with coxsackievirus B3 myocarditis and endomyocardial biopsies from patients with myocarditis and dilated cardiomyopathy. Jpn Circ J 56: 1081-1093.

6. Ukimura A, Satomi H, Ooi Y, Kanzaki Y (2012) Myocarditis Associated with Influenza A H1N1pdm2009. Influenza Res Treat 2012: 351979.

7. Bratincsák A, El-Said HG, Bradley JS, Shayan K, Grossfeld PD, et al. (2010) Fulminant myocarditis associated with pandemic H1N1 influenza A virus in children. J Am Coll Cardiol 55: 928-929.

8. Aoyama N, Izumi T, Hiramori K, Isobe M, Kawana M, et al. (2002) National survey of fulminant myocarditis in Japan: therapeutic guidelines and long-term prognosis of using percutaneous cardiopulmonary support for fulminant myocarditis (special report from a scientific committee). Circ J 66: 133-144.

9. Centers for Disease Control and Prevention (CDC) (2010) Patients hospitalized with 2009 pandemic influenza A (H1N1) - New York City, May 2009. MMWR Morb Mortal Wkly Rep 58: 1436-1440.

10. Van Kerkhove MD, Vandemaele KA, Shinde V, Jaramillo-Gutierrez G, Koukounari A, et al. (2011) Risk factors for severe outcomes following 2009 influenza A (H1N1) infection: a global pooled analysis. PLoS Med 8: e1001053.

11. Weiss TW, Stensaeth KH, Eritsland J (2010) Myocarditis in a juvenile patient with influenza A virus infection. Eur Heart J 31: 277.

12. Ukimura A, Izumi T, Matsumori A, Clinical Research Committee on Myocarditis Associated with the 2009 Influenza A (H1N1) Pandemic in Japan organized by the Japanese Circulation Society (2010) A national 
Citation: Ukimura A, Ooi Y, Kanzaki Y (2015) The Neuraminidase Inhibitor Peramivir Ameliorates Myocarditis Induced by Influenza A (H1N1pdm) Virus in a Murine Model. Clin Microbiol 4: 201. doi:10.4172/2327-5073.1000201

Page 5 of 5

survey on myocarditis associated with the 2009 influenza A (H1N1) pandemic in Japan. Circ J 74: 2193-2199.

13. Ukimura A, Ooi Y, Kanzaki Y, Inomata T, Izumi T (2013) A national survey on myocarditis associated with influenza H1N1pdm2009 in the pandemic and postpandemic season in Japan. J Infect Chemother 19: 426-431.

14. Ukimura A, Kishi K, Yamada T, Shibata Y, Ooi Y, et al. (2014) National Survey of Influenza Myocarditis in Japanese Children in Three Seasons. Clinical Microbiol 3: 143.

15. Rezkalla SH, Kloner RA (1989) Management strategies in viral myocarditis. Am Heart J 117: 706-708.

16. Meune C, Spaulding C, Mahé I, Lebon P, Bergmann JF (2003) Risks versus benefits of NSAIDs including aspirin in myocarditis: a review of the evidence from animal studies. Drug Saf 26: 975-981.

17. Bantia S, Parker CD, Ananth SL, Horn LL, Andries K, et al. (2001) Comparison of the anti-influenza virus activity of RWJ-270201 with those of oseltamivir and zanamivir. Antimicrob Agents Chemother 45: 1162-1167.

18. Kohno S, Kida H, Mizuguchi M, Shimada J; S-021812 Clinical Study Group (2010) Efficacy and safety of intravenous peramivir for treatment of seasonal influenza virus infection. Antimicrob Agents Chemother 54: 4568-4574.

19. Bantia S, Kellogg D, Parker C, Upshaw R, Ilyushina NA, et al. (2011) A single intramuscular injection of neuraminidase inhibitor peramivir demonstrates antiviral activity against novel pandemic A/California/ 04/2009 (H1N1) influenza virus infection in mice. Antiviral Res 90: 17-21.

20. Kodama M, Yoshida R, Hasegawa T, Izawa M, Kitano M, et al. (2014) The relationship between in vivo antiviral activity and pharmacokinetic parameters of peramivir in influenza virus infection model in mice. Antiviral Res 109: 110-115.

21. Sugaya N (2011) Widespread use of neuraminidase inhibitors in Japan. J Infect Chemother 17: 595-601.

22. Sugaya N, Shinjoh M, Mitamura K, Takahashi T (2011) Very low pandemic influenza A (H1N1) 2009 mortality associated with early neuraminidase inhibitor treatment in Japan: analysis of 1000 hospitalized children. J Infect 63: 288-294.

23. Ikematsu H, Kawai N, Iwaki N, Kashiwagi S (2014) In vitro neuraminidase inhibitory activities of four neuraminidase inhibitors against influenza viruses isolated in the 2011-2012 season in Japan. J Infect Chemother 20: 77-80.

24. Jefferson T, Jones MA, Doshi P, Del Mar CB, Hama R, et al (2004) Neuraminidase inhibitors for preventing and treating influenza in healthy adults and children. Cochrane Database Syst Rev 4.

25. The Japanese Association for Infectious Diseases (2009) Guidelines for diagnosis and treatment of pandemic influenza.
26. Japanese Ministry of Health, Labour and Welfare (2014) Guidelines for treatment of pandemic influenza in adults.

27. Hama R, Jones M, Okushima H, Kitao M, Noda N, et al. (2011) Oseltamivir and early deterioration leading to death: a proportional mortality study for 2009A/H1N1 influenza. Int J Risk Saf Med 23: 201-215

28. Japanese summary basis of approval (SBA) of peramivir.

29. Tanaka A, Nakamura S, Seki M, Iwanaga N, Kajihara T, et al. (2015) The effect of intravenous peramivir, compared with oral oseltamivir, on the outcome of post-influenza pneumococcal pneumonia in mice. Antivir Ther 20: 11-19.

30. Wong ZX, Jones JE, Anderson GP, Gualano RC (2011) Oseltamivir treatment of mice before or after mild influenza infection reduced cellular and cytokine inflammation in the lung. Influenza Other Respir Viruses 5: 343-350.

31. Gualano RC, Hansen MJ, Vlahos R, Jones JE, Park-Jones RA, et al. (2008) Cigarette smoke worsens lung inflammation and impairs resolution of influenza infection in mice. Respir Res 9: 53.

32. Pan HY, Yamada H, Chida J, Wang S, Yano M, et al. (2011) Upregulation of ectopic trypsins in the myocardium by influenza A virus infection triggers acute myocarditis. Cardiovasc Res 89: 595-603.

33. Kotaka M, Kitaura Y, Deguchi H, Kawamura K (1990) Experimental influenza A virus myocarditis in mice. Light and electron microscopic, virologic, and hemodynamic study. Am J Pathol 136: 409-419.

34. Kishimoto C, Hiraoka Y, Takada H (2004) Effects of immunoglobulin upon murine myocarditis caused by influenza A virus: superiority of intact type to $\mathrm{F}(\mathrm{ab}) 2$ type. J Cardiovasc Pharmacol 43: 61-67.

35. Pan HY, Yano M, Kido H (2011) Effects of inhibitors of Toll-like receptors, protease-activated receptor-2 signalings and trypsin on influenza A virus replication and upregulation of cellular factors in cardiomyocytes. J Med Invest 58: 19-28.

36. Hayden FG, Treanor JJ, Fritz RS, Lobo M, Betts RF, et al. (1999) Use of the oral neuraminidase inhibitor oseltamivir in experimental human influenza: randomized controlled trials for prevention and treatment. JAMA 282: 1240-1246.

37. Crain SM, Shen KF (2004) Neuraminidase inhibitor, oseltamivir blocks GM1 ganglioside-regulated excitatory opioid receptor-mediated hyperalgesia, enhances opioid analgesia and attenuates tolerance in mice. Brain Res 995: 260-266.

38. Moore ML, Chi MH, Zhou W, Goleniewska K, O'Neal JF, et al. (2007) Cutting edge: oseltamivir decreases T cell GM1 expression and inhibits clearance of respiratory syncytial virus: potential role of endogenous sialidase in antiviral immunity. J Immunol 178: 2651-4265. 